\title{
Baseband and RF hardware impairments in full-duplex wireless systems: experimental characterisation and suppression
}

\author{
Alexios Balatsoukas-Stimming*, Andrew CM Austin, Pavle Belanovic and Andreas Burg
}

\begin{abstract}
Hardware imperfections can significantly reduce the performance of full-duplex wireless systems by introducing non-idealities and random effects that make it challenging to fully suppress self-interference. Previous research has mostly focused on analysing the impact of hardware imperfections on full-duplex systems, based on simulations and theoretical models. In this paper, we follow a measurement-based approach to experimentally identify and isolate these hardware imperfections leading to residual self-interference in full-duplex nodes. Our measurements show the important role of images arising from in-phase and quadrature $(\mathrm{IQ})$ imbalance in the transmitter and receiver mixers. We also observe baseband non-linearities in the digital-to-analog converters (DAC), which can introduce strong harmonic components in the transmitted signal that have not been considered previously. A corresponding general mathematical model to suppress these components of the self-interference signal arising from the hardware non-idealities is developed from the observations and measurements. Results from a $10 \mathrm{MHz}$ bandwidth full-duplex system, operating at $2.48 \mathrm{GHz}$, show that up to $13 \mathrm{~dB}$ additional suppression, relative to state-of-the-art implementations, can be achieved by jointly compensating for IQ imbalance and DAC non-linearities.
\end{abstract}

Keywords: Full-duplex communications; Transceiver non-idealities; Digital self-interference cancellation

\section{Introduction}

The increasing demand for wireless communications has renewed interest in developing systems that use the limited radio spectrum more efficiently to increase throughput, support additional users and improve capacity. A promising physical layer approach to increase the spectral utilisation is full-duplex operation, where transceivers in the system transmit and receive simultaneously in the same frequency band [1-3]. It is important to note that most contemporary wireless communication systems use half duplex, separating transmission and reception in frequency or time. Accordingly, full-duplex systems can potentially double the spectral efficiency $[2,4,5]$ and are of considerable interest for cognitive radio [6,7], cooperative communications $[8,9]$, relay networks $[10,11]$ and $5 \mathrm{G}$ wireless systems [3]. Furthermore, full-duplex links can be used to increase physical layer secrecy [12],

*Correspondence: alexios.balatsoukas@epfl.ch

Telecommunications Circuits Laboratory, EPFL, STI-IEL-TCL, Station 11, $\mathrm{CH}-1015$ Lausanne, Switzerland improve medium access control (MAC) layer protocols to increase throughput [13] and simplify resource allocation and spectrum management [2].

One of the main challenges for realising full-duplex systems is the presence of strong self-interference arising from physically close transmitting and receiving antennas $[1,4,5]$. A full-duplex system can also be implemented using only a single antenna and a circulator; however, imperfect isolation will still allow self-interference signals to leak between the transmit and receive circuits. The power of the self-interference signal can be orders of magnitude larger than any external signal of interest, and full-duplex systems must therefore implement self-interference suppression [2]. Ideally, this suppression will reduce the power of the self-interference signal to or below the noise floor. Where this cannot be achieved, the residual self-interference will lower the signal-to-noise ratio (SNR) and reduce throughput.

In principle, perfectly suppressing the self-interference signal should be possible, as the baseband transmitted

\section{量 Springer}

(C) 2015 Balatsoukas-Stimming et al. This is an Open Access article distributed under the terms of the Creative Commons Attribution License (http://creativecommons.org/licenses/by/4.0), which permits unrestricted use, distribution, and reproduction in any medium, provided the original work is properly credited. 
signal is always known within the full-duplex node. However, previous research has shown that the actual selfinterference signal is a complicated function of the baseband transmitted signal, which is altered through many effects that are only partially understood or known [2]. In addition, non-idealities in the receiver circuitry can further distort the signal prior to digital self-interference cancellation. The impact and mitigation of transmitter and receiver effects have been considered extensively in the literature for conventional wireless communication systems, for example, [14-16]. However, full-duplex systems require a more detailed characterisation and modelling of these non-idealities due to the considerable power of the self-interference signal compared to the desired signal. Hence, transceiver hardware imperfections are still a significant, if not the dominant limiting factor for analog and digital self-interference suppression techniques $[2,7]$. Furthermore, the relative significance of each hardware imperfection (or combinations thereof) depends on the device characteristics and the specific system implementation and configuration.

On the transmitter side, oscillator phase noise [17-20] and in-phase and quadrature (IQ) imbalance introduced by the mixers $[21,22]$ and non-linearities introduced by RF power amplifiers [21-25] have all been shown to reduce the amount of analog and digital self-interference suppression that can be achieved. A number of approaches to mitigate some of these hardware effects have been proposed for full-duplex systems, for example, [21] developed a digital domain scheme to compensate the IQ imbalance introduced by mixers, while [17-20] showed that sharing the oscillator between the transmit, receive and cancellation chains can reduce the impact of phase noise. It should be noted that some hardware components and resulting impairments - such as oscillator phase noise and mixer IQ imbalance - are common to both transmitter and receiver circuits. Previous research on receiver-specific hardware impairments has largely focused on the impact of imperfect analog-to-digital converters (ADC) [21,26,27]. In particular, [21] examined the quantisation noise introduced by the ADCs, while [26] examined the impact of limited $\mathrm{ADC}$ resolution and established the relationships between the ADC resolution and the required analog suppression to achieve a sufficient signal to self-interference ratio. The impact and mitigation of sampling jitter on self-interference suppression was considered in $[28,29]$. However, most of these studies are based on theoretical models and simulation analysis of assumed hardware imperfections without experimental verification. Unfortunately, it is mostly the uncertainty and inaccuracy of the models that renders the suppression of these effects difficult. Hence, their identification through measurements and experiments takes an important role in enabling fullduplex communication nodes.
Contributions and outline: The motivation for this paper is, therefore, to consider an experimental characterisation of the hardware imperfections present on a full-duplex platform to complement the existing body of theoretical studies describing their impact. Specifically, we confirm the existence and the importance of most transmitter impairments that have been previously studied theoretically in the literature. More importantly, we identify a strong baseband non-linearity coming from the digitalto-analog converters (DACs) which, to the best of our knowledge, has not received any attention in the existing full-duplex literature. This paper also addresses hardware impairments introduced by the receiver oscillator and mixers, but it does not include other receiver side effects, such as non-linearities introduced by the ADC. While the measured values for the impairments are specific to our hardware platform, the findings are generalised to a parametric cancellation model that can be applied to any similar full-duplex system. This digital cancellation model jointly takes into account DAC non-linearities and IQ imbalance and outperforms existing digital cancellation methods.

The paper is organised as follows. Section 2 outlines the various self-interference suppression architectures and their susceptibility to hardware imperfections. A brief description of our hardware testbed is provided in Section 3. In Section 4, we measure and model the nonideal effects introduced by the hardware using single tone tests. Section 5 shows the impact of correctly compensating for the hardware effects identified in the previous sections for a wideband full-duplex transceiver.

\section{Self-interference suppression in full-duplex wireless systems}

Physical separation and inherent attenuation between the transmit and receive circuits introduces a small amount of passive suppression. However, typically passive suppression (or the isolation through a circulator) alone is insufficient to allow reliable full-duplex communications [30]. Additional signal processing is required to actively suppress and reduce the strength of the self-interference to the noise floor. In general, some of this active suppression must be achieved before the received signal is digitised, i.e., in the analog domain, since entirely digital suppression is usually not feasible due to limited ADC resolution when the external signal-of-interest is small relative to the self-interference [2].

For example, Figure 1 shows the required suppression budget for a $10 \mathrm{MHz}$ full-duplex wireless system, with a maximum transmit power of $20 \mathrm{dBm}$. For this operating bandwidth, the typical measured noise floor is approximately $-90 \mathrm{dBm}$. The peak-to-average power ratio (PAPR) of the transmitted signal is assumed to be $10 \mathrm{~dB}$, and we must therefore allow a similar amount of 


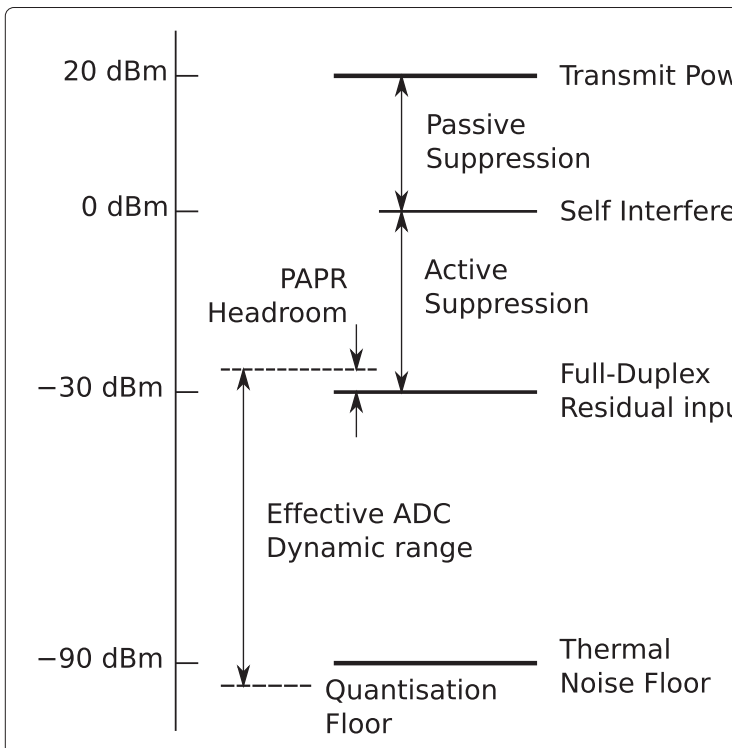

Figure 1 Power budget for a full-duplex wireless system showing the various stages required to suppress the transmitted signal.

head room to avoid overloading the receiver front-end circuitry. We note that the effective number of bits (ENOB) of the ADC that is used in our system is 11.5 bits [31]. Thus, its dynamic range is approximately equal to $70 \mathrm{~dB}$, which is the number we used in the budget of Figure 1. To prevent the system from being limited by the ADC quantisation, it is necessary to place the quantisation floor at or below the thermal noise floor. At $20 \mathrm{dBm}$ transmit power, a minimum of $50 \mathrm{~dB}$ suppression is required from the combined passive and analog suppression stages to reduce the power of the self-interference to a level where the remaining signal can be captured within the dynamic range of the ADC, with a sufficient resolution for the desired signal after digital domain suppression of the residual self-interference.

\subsection{Active analog suppression}

One of the most widely considered and successful active analog suppression techniques is to subtract a cancellation signal from the received signal (which contains the self-interference and any desired signal) $[1,4,5]$. A number of techniques for generating this cancellation signal have been proposed; however, these generally fall into two categories.

The Stanford architecture [4,32] proposes measuring the transmitted RF signal (e.g., with a low insertion loss coupler) immediately before it is applied to the antenna. The cancellation signal is generated by appropriately delaying and attenuating this measured signal to account for propagation in the real self-interference channel. A practical implementation of this approach using tapped delay lines achieved $45 \mathrm{~dB}$ active suppression, over a $40 \mathrm{MHz}$ bandwidth at $2.45 \mathrm{GHz}$ [4]. An advantage of the Stanford architecture is that hardware imperfections introduced by the transmitter circuit are inherently included in the cancellation signal. However, systemspecific RF cancellation circuits are required to model the effect of the channel and the architecture does not scale well to multiple-input multiple-output (MIMO) systems, as each transmitter-receiver pair requires a dedicated circuit [32].

In contrast, the analog cancellation signal in the Rice architecture is generated using a separate RF chain $[5,33]$. The baseband input to the cancellation chain is computed by sounding the self-interference channel to determine the appropriate delay, attenuation, and distortion parameters that must be applied. Several implementations of the Rice architecture have been reported [5,34]. The initial prototype achieved between 20 and $34 \mathrm{~dB}$ analog suppression for transmit powers ranging from 0 and $15 \mathrm{dBm}$ (generally suppression decreases with increasing transmit power), over a $40 \mathrm{MHz}$ bandwidth on a WARP platform [5]. Similar results were reported using a National Instruments (NI) FlexRIO platform, with an average $48 \mathrm{~dB}$ active suppression over a $20 \mathrm{MHz}$ bandwidth for $4 \mathrm{dBm}$ transmit power [34]. For both implementations, further suppression was achieved in the digital domain; however, both groups observed the suppression introduced by digital cancellation depended on the analog stage and that the total suppression achieved was approximately constant $[5,18,34]$. The Rice architecture can be more readily extended to MIMO systems (as each receiver only requires one additional RF cancellation chain) and does not require specialised RF hardware design. However, it is important to note that hardware imperfections are potentially more prevalent in the Rice architecture, as the cancellation signal is generated from a separate RF chain.

\subsection{Active digital suppression}

The active analog suppression stage, which is usually not perfect, can be followed by suppression in the digital domain to further remove remaining self-interference. In general, i.e., with or without active analog suppression, the received complex baseband signal, $r$, can be expressed as a sum of: the external signal-of-interest, $s$; a function, $f(\cdot)$ of the complex baseband transmitted self-interference signal, $x$; and noise, $z$. The goal of digital suppression is to estimate $f(\cdot)$ and subtract $f(x)$ from the received signal.

Since the transmitted signal is distorted by transmitter non-idealities, the self-interference signal is a complicated function of $x$. Sophisticated digital cancellation methods are required to capture those transmitter imperfections $[24,25]$. A thorough identification and characterisation of these non-idealities is presented in Section 4, and digital cancellation schemes are presented in Section 5. 


\section{System architecture and testbed setup}

Our full-duplex node testbed (running the LabVIEW software platform) consists of an NI FlexRIO PXIe-1082 chassis [35] with two NI 5791R RF transceiver adapter modules [36], each containing one transmitter and one receiver. Each NI 5791R module inherently uses the same oscillator for the receiver and the transmitter, and the carrier signal can also be shared between multiple cards. The NI 5791R operates at carrier frequencies of $400 \mathrm{MHz}$ up to $4.4 \mathrm{GHz}$ and provides peak output powers ranging from -24 to $8 \mathrm{dBm}$. In order to be able to test realistic output powers of up to $22 \mathrm{dBm}$, we use an external amplifier [37] that provides $14 \mathrm{~dB}$ of gain. A photograph of the system is shown in Figure 2.

The digital baseband transmit samples are generated in MATLAB and sent to the testbed over a network. The testbed is responsible for synchronising the receiver and transmitter and handles all analog frontend tasks (i.e., digital-to-analog conversion, mixing, amplification, RF filtering and analog-to-digital conversion). The received digital baseband samples are then sent back to MAT$\mathrm{LAB}$ for offline processing. For the measurements in this paper, we use an RF frontend that consists of two $2.4 \mathrm{GHz}$ antennas with variable spacing and orientation.

In this paper, we use this two-antenna configuration to emulate the analog suppression by increasing the level of passive isolation. While our analog suppression achieves the necessary $50 \mathrm{~dB}$ attenuation even with a circulator, we are interested in observing all signal components in the digital domain for our measurements and experiments in order to enable a coherent illustration and interpretation of the results. Hence, in the following, we use the system with the active analog suppression chain deactivated.

\section{Transceiver impairments}

In this section, we discuss the main sources of nonidealities in the self-interference signal and we provide measurements from our testbed that clearly demonstrate the existence of most of the impairments that have been previously considered in the bibliography. More specifically, we confirm the presence and the effect of phase noise [17-20], IQ imbalance [21,22] and RF non-linearities $[22,24,25]$. More importantly, however, we observe the existence of baseband non-linearities with significant power. We show that these non-linearities are highly consistent with non-linearities stemming for the transmitter DACs, and we provide a corresponding DAC non-linearity model that can be used for digital selfinterference cancellation.

We denote the discrete time digital baseband signal by $x[n]$ and the continuous time analog baseband signal by $\tilde{x}(t)$. The upconverted analog signal is denoted by $x(t)$, and the amplified upconverted analog signal is denoted by $\hat{x}(t)$. The downconverted analog self-interference signal at the receiver is denoted by $\tilde{r}(t)$, and the digital baseband self-interference signal is denoted by $r[n]$. For simplicity, we omit the time indices $n$ and $t$, unless strictly necessary (e.g., to denote a delay as in the case of phase noise), as they will always be clear from the context. Figure 3 shows a block diagram of a full-duplex transceiver node, with all important analog front-end components and signal terms identified.

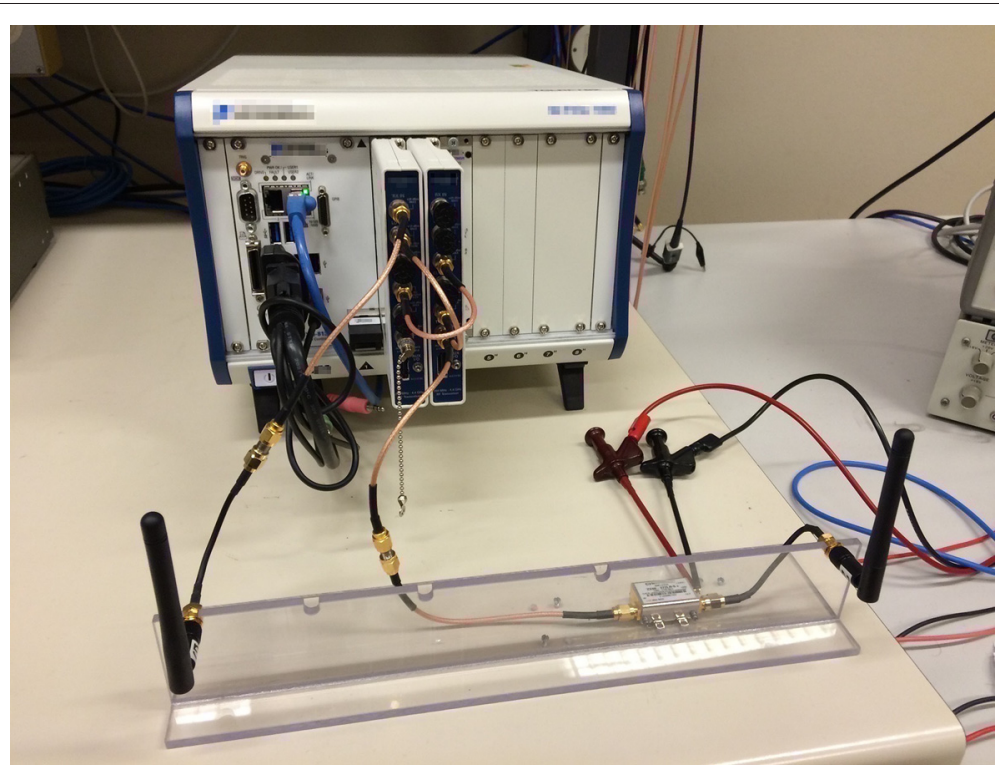

Figure 2 Full-duplex front-end. Showing the antenna configuration considered and the NI FlexRIO hardware platform with two NI 5791 R RF transceiver adapter modules installed. 


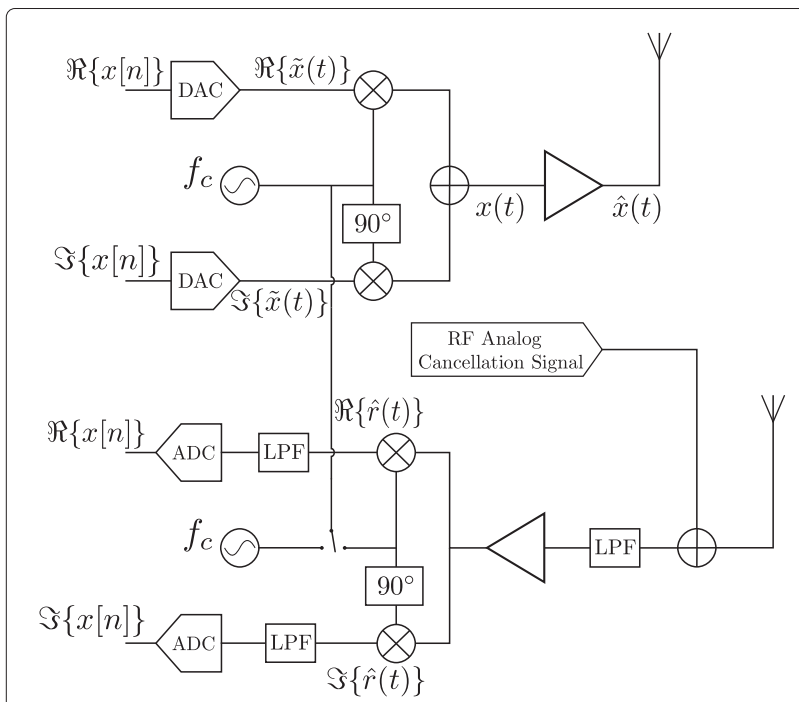

Figure 3 Block diagram of a full-duplex transceiver node with all important analog front-end components.

\subsection{Phase noise}

The upconversion of the baseband signal to the carrier frequency $f_{c}$ is performed at the transmitter by mixing the baseband signal with a carrier signal. The oscillators that are used to generate the carrier signal suffer from various impairments, the most significant of which is phase noise. Thus, instead of generating a pure tone at frequency $f_{c}$, i.e., $e^{j 2 \pi f_{c} t}$, the generated tone is actually $e^{j\left(2 \pi f_{c} t+\phi(t)\right)}$, where $\phi(t)$ is the random phase noise process. The downcoversion process at the receiver is also affected by phase noise, since it uses a similarly generated carrier signal.

The effect of phase noise on full-duplex transceivers has been extensively studied [18-20]. In order to summarise and illustrate the effect of the phase noise, we assume for the moment that all parts of the transceiver are ideal, except for the oscillators that generate the carrier signal. Moreover, for illustration purposes, the self-interference channel is assumed to introduce a simple delay, i.e., it can be represented as $\delta(t-\Delta t)$. Such a delay can for example arise from acoustic wave bandpass filters in the receive chain. At the transmitter, phase noise is introduced during the upconversion process, so the transmitted RF signal is:

$$
\hat{x}=\Re\left\{\tilde{x} e^{j\left(2 \pi f_{c} t+\phi_{\mathrm{Tx}}(t)\right)}\right\},
$$

where $\phi_{\mathrm{Tx}}(t)$ denotes the phase noise process of the oscillator used by the transmitter. At the receiver, phase noise is introduced during the downconversion process:

$$
\begin{aligned}
\hat{r} & =\operatorname{LPF}\left\{\hat{x} e^{-j\left(2 \pi f_{c} t+\phi_{\mathrm{Rx}}(t-\Delta t)\right)}\right\} \\
& =\tilde{x} e^{j\left(\phi_{\mathrm{Tx}}(t)-\phi_{\mathrm{Rx}}(t-\Delta t)\right)-2 \pi f_{c} \Delta t}
\end{aligned}
$$

where LPF denotes a low-pass filter that removes the copies of the signal around $2 f_{c}$ and $-2 f_{c}$ and $\phi_{\mathrm{Rx}}(t)$ denotes the phase noise process of the oscillator used by the receiver. If the transmitter and the receiver use independent oscillators, the $\phi_{\mathrm{Tx}}(t)$ and $\phi_{\mathrm{Rx}}(t)$ processes will be uncorrelated. However, in full-duplex transceivers, the transmitter and the receiver are typically co-located and can physically share the same oscillator. Thus, $\phi_{\mathrm{Tx}}(t)=$ $\phi_{\mathrm{Rx}}(t)$, and we denote the common phase noise process by $\phi(t)$. Due to the delay introduced by the transmission channel, the phase noise instances experienced by the signal at the transmitter and the receiver mixer are not identical. However, it is evident that if the delay is such that $\phi(t)$ and $\phi(t-\Delta t)$ are highly correlated, then sharing the oscillator can significantly reduce the effect of phase noise in the received signal after the mixer [18].

In order to demonstrate the improvement obtained by sharing the oscillator between the transmitter and the receiver mixers, we perform a one-tone test on our testbed and we examine the received self-interference signal. We use the lowest possible transmit power setting (i.e., $-10 \mathrm{dBm}$ ) in order to minimise non-linearities arising from the amplifier of the transmitter. An indicative spectrum of the received self-interference signal is presented in Figure 4a. We observe that the received signal has significant spectral content around the transmitted tone, indicated by (i), with the most powerful components lying approximately $46 \mathrm{~dB}$ below the power of the tone. We note that the two independent oscillators use the same $10 \mathrm{MHz}$ reference signal, but this is not sufficient to reduce the effect of phase noise, which is caused by the phase-locked loop (PLL) that generates the actual carrier signal from the reference signal. We also observe numerous tones arising from other non-linearities, which will be explained in the following sections. In Figure 4b, we present an indicative spectrum of the received selfinterference signal when the transmit and receiver mixers use the same oscillator. In this case, the strongest component of the spectral content resulting from phase noise lies approximately $70 \mathrm{~dB}$ below the received tone. We observe that the phase noise induced noise floor will lie significantly below the noise floor introduced by the remaining non-linearities.

\subsection{Baseband non-linearities}

In Figure $4 \mathrm{a}$, we observe that numerous tones have appeared in the received signal apart from the transmitted tone. The eminent tones on the positive side of the spectrum appear at integer multiples of the transmitted tone frequency. Since the transmitter amplifier is set to its lowest possible setting and, more importantly, we observe even harmonics of the transmitted tone, we can safely 


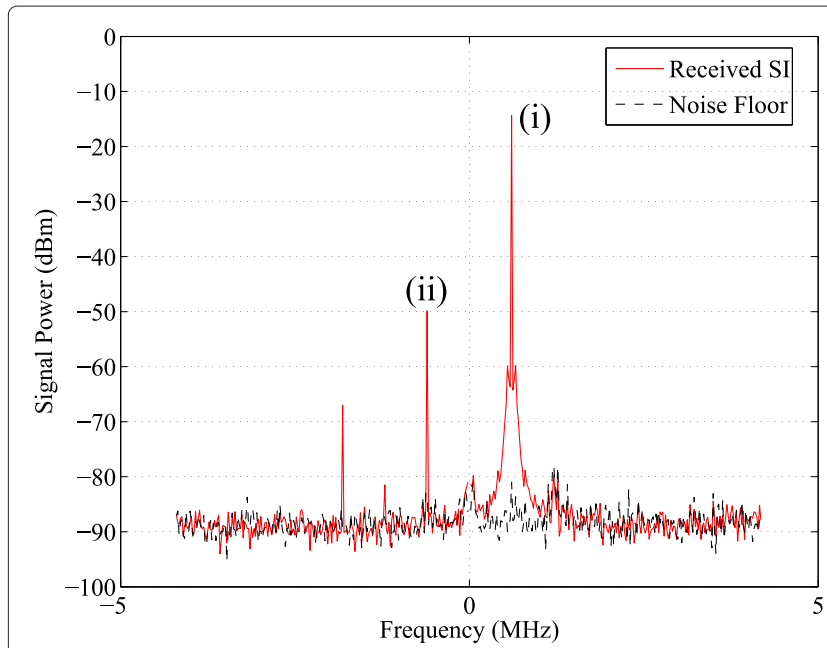

(a)

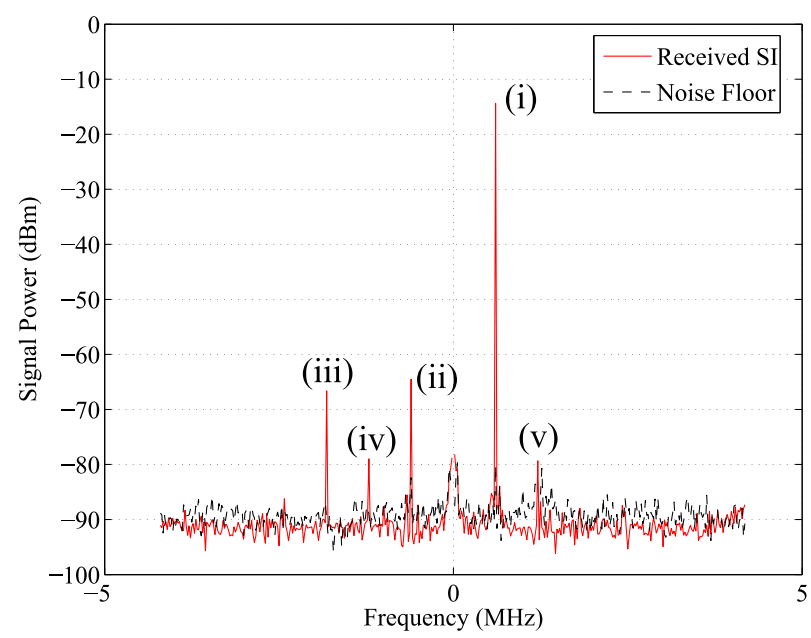

(c)

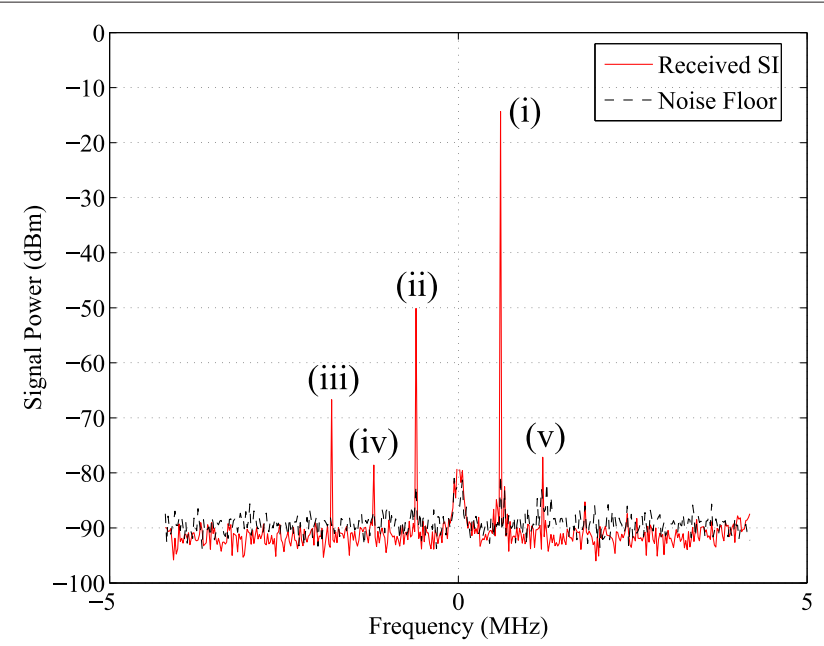

(b)

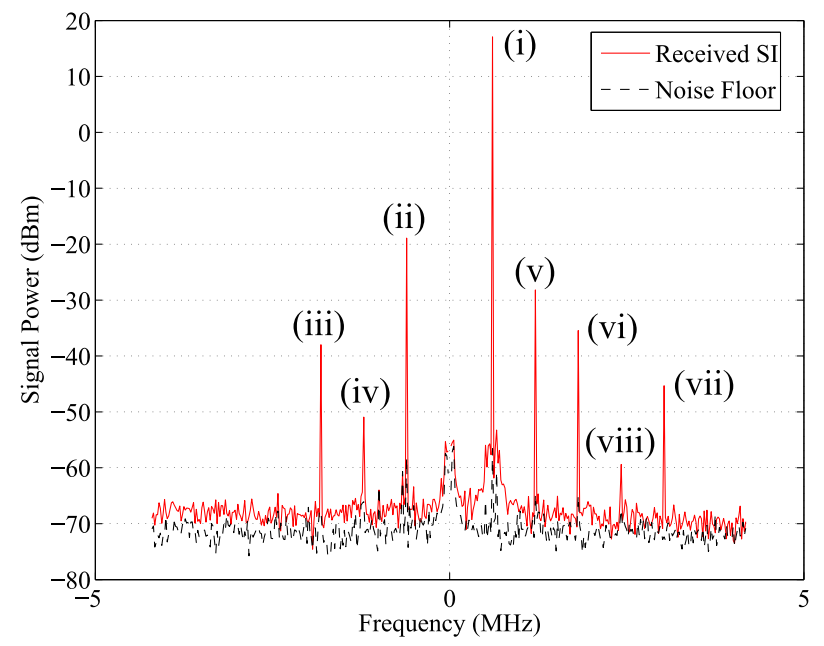

(d)

Figure 4 Received self interference signals with specific tones identified. (a) - $10 \mathrm{dBm}$ transmit power and independent oscillators. (b) - $10 \mathrm{dBm}$ and shared oscillators. (c) $-10 \mathrm{dBm}$, shared oscillators and IQ imbalance compensation.s (d) $+20 \mathrm{dBm}$ transmit power, shared oscillator and IQ imbalance compensation.

conclude that these harmonics must (at least partially) occur in the analog baseband signal. The only components that can introduce non-linearities in the transmitter side analog baseband signal are the two DACs. On the receiver side, we have two ADCs, which can also introduce non-linearities in the observed digital baseband signal. However, the ADC used in the NI 5791R transceiver [31] has a higher spurious-free dynamic range (SFDR) than the DAC [38], so we assume that all baseband non-linearities stem from the DACs.

We model the DAC-induced non-linearities by using a Taylor series expansion around 0 of maximum degree $m_{\max }$. In the block diagram of Figure 3 , we see that the first DAC has $\Re\{x\}$ as its input and the second DAC has $\Im\{x\}$ as its input. Thus the output signal of each DAC can be written as:

$$
\begin{aligned}
& \Re\{\tilde{x}\}=\sum_{m=1}^{m_{\max }} \alpha_{1, m} \Re\{x\}^{m}, \\
& \Im\{\tilde{x}\}=\sum_{m=1}^{m_{\max }} \alpha_{2, m} \Im\{x\}^{m},
\end{aligned}
$$

where $\alpha_{i, m} \in \mathbb{R}, i \in\{1,2\}, m \in\left\{1, \ldots, m_{\max }\right\}$. Thus, the continuous time complex baseband signal $\tilde{x}$ can be written as:

$$
\tilde{x}=\sum_{m=1}^{m_{\max }} \alpha_{1, m} \Re\{x\}^{m}+j \sum_{m=1}^{m_{\max }} \alpha_{2, m} \Im\{x\}^{m} .
$$

By analysing Equation 6 with a single input tone of frequency $f$, it can be shown that, if the DACs are perfectly matched so that $\alpha_{1, m}=\alpha_{2, m}, m \in\left\{1, \ldots, m_{\max }\right\}$, the 
DAC induced non-linearities produce harmonics alternating on only one side of the spectrum for odd $m$, but on both sides of spectrum for even $m$. More specifically, it is shown in the appendix that for odd $m$, we obtain harmonics at frequencies $m(-1)^{\frac{m-1}{2}} f$, while for even $m$, we obtain harmonics at both $-m f$ and $m f$ with equal power. We observe in Figure $4 \mathrm{~b}$ that the frequency $3 f$ is not present but the frequency $-3 f$, indicated by (iii), is present and also that the harmonics at $-2 f$ and $2 f$ (indicated by (iv) and (v), respectively) have approximately equal power. Thus, all our observations are in complete agreement with what we expect to see based on our model and the proof in the appendix. Tone (ii), at frequency $-f$, which we observe clearly in Figure $4 \mathrm{~b}$ but is not predicted by the DAC nonlinearities, is the result of IQ imbalance, as we will explain in the following section.

It is interesting to note in Figure $4 \mathrm{~b}$ that tone (iii), at $-3 f$, is stronger than tone (ii), at $-2 f$, which seems counterintuitive at first. However, when downscaling the digital baseband signal, we observe that the power of the third harmonic decreases at a higher rate than the power of the second harmonic, which is consistent with what one would expect.

\subsection{IQ imbalance}

IQ imbalance is caused by amplitude and phase mismatch in the in-phase and the quadrature components of the upconverted analog signal. To simplify notation, in this section, we consider frequency-flat IQ imbalance. The output of the non-ideal mixer can be modelled as:

$$
x=\Re\left\{\left(\gamma_{\mathrm{T} \mathrm{x}} \tilde{x}+\delta_{\mathrm{Tx}} \tilde{x}^{*}\right) e^{j 2 \pi f_{c} t}\right\},
$$

where $\gamma_{\mathrm{Tx}}, \delta_{\mathrm{Tx}} \in \mathbb{C}$. We note that any amplitude mismatch in the linear components of the DACs will also manifest itself as IQ imbalance.

In Figure 4a, we observe that there exists a mirror image of the transmitted tone, with respect to the carrier frequency at frequency $-f$ (indicated by (ii)) which arises due to the effect of IQ imbalance. However, it is important to note that the signal components that - at first sight - appear to be harmonics of this negative tone instead can only arise due to the DAC non-linearities as explained earlier. This is for several reasons: first, the harmonic of the original tone $f$ at frequency $3 f$ (not indicated in the figure as it lies below the thermal noise floor) is significantly weaker than the alleged harmonic of $-f$ at frequency $-3 f$ (indicated by (iii)). Moreover, since there are no significant baseband non-linearities after the mixer of the receiver, we do not expect to observe a second harmonic of $-f$ at frequency $-2 f$. Finally, when we enable the built-in IQ imbalance compensation block of the NI 5791R transceivers, as demonstrated in Figure 4c, we observe that, while the power of the IQ imbalance induced tone (ii), at $-f$, is reduced by approximately $20 \mathrm{~dB}$, the apparent harmonics of this tone at $-2 f$ and $-3 f$, indicated by (iii) and (iv), respectively, are unaffected. In order to have IQ imbalance that is similar to what a low-cost transceiver would experience, we keep the built-in IQ imbalance compensation mechanism of the NI 5791R disabled.

\subsection{RF non-linearities}

Non-linearities in the upconverted RF signal are caused by the power amplifier that comes after the RF mixer. These non-linearities mainly appear when the amplifier is operated in its non-linear region, i.e., close to its maximum output power, where significant compression of the output signal occurs. Basic arithmetic manipulations can show that all the even-power harmonics lie out of band and will be cutoff by the RF low-pass filter of the receiver. The RF non-linearities can be modelled using a Taylor series expansion around 0 of maximum degree $n_{\max }$ :

$$
\hat{x}=\sum_{\substack{n=1, n \text { odd }}}^{n_{\max }} \beta_{n} x^{n},
$$

where $\beta_{n} \in \mathbb{R}, n \in\left\{1,3, \ldots, n_{\max }\right\}$.

The effect of RF non-linearities can be clearly seen in Figure $4 \mathrm{~d}$, where we present the spectrum of the received self-interference signal when transmitting with an output power of $20 \mathrm{dBm}$. We observe that strong third and fifth harmonics of the transmitted tone $f$ appear (indicated by (vi) and (vii), respectively). The power of the tones on the negative frequencies with respect to the main tone $f$ remains almost unaffected, as expected because they do not arise from the RF non-linearities, but from the DAC non-linearities. The third and fifth harmonics of the tones on the negative frequencies lie below the noise floor. We also observe that the noise floor has increased by $20 \mathrm{~dB}$. This is caused by the effect of the limited dynamic range of the ADCs of the receiver, which means that quantisation noise dominates thermal noise. As can be seen by referring to the power budget in Figure 1, we would require at least $20 \mathrm{~dB}$ more passive or active analog suppression in order to observe the thermal noise floor.

\section{Digitally cancelling the non-idealities}

As mentioned earlier, the goal of digital cancellation is to reconstruct the self-interference signal (including all transmitter non-idealities) and subtract it from the received signal. In this section, we briefly describe existing digital cancellation methods that take into account some of the transmitter impairments and we describe our proposed joint digital cancellation scheme. 


\subsection{Existing digital cancellation methods}

In general, i.e., with or without active analog suppression, the received complex baseband signal $r$ can be written as:

$$
r=f(x)+s+z
$$

where $f(x)$ denotes a function of the complex baseband self-interference signal $x, s$ denotes the signal-of-interest and $z$ denotes thermal noise. The goal of digital suppression is to estimate the function $f$ and subtract $f(x)$ from the received signal. In order to do so, $f$ needs to be modelled in some way.

The simplest form of digital cancellation, called linear cancellation, models $f$ as a convolution with the selfinterference channel, denoted by $h_{\mathrm{SI}}$, i.e.,

$$
r=h_{\mathrm{SI}} * x+s+z,
$$

where $*$ denotes the convolution operation. By writing the convolution as a matrix operation, a least squares (LS) estimate for $h_{\mathrm{SI}}$ can be obtained, which we denote by $\hat{h}_{\mathrm{SI}}$. Linear digital cancellation can then be written as:

$$
r-\hat{r}=h_{\mathrm{SI}} * x-\hat{h}_{\mathrm{SI}} * x+s+n,
$$

In order to capture amplifier induced non-linearities, the non-linear digital cancellation proposed in [24] assumes that:

$$
r=\sum_{\substack{n=1, n \text { odd }}}^{n_{\max }} h_{\mathrm{SI}, n} * x^{n}+s+z,
$$

where $h_{\mathrm{SI}, n}, n=1,3, \ldots, n_{\max }$, denotes the selfinterference channel experienced by each of the harmonics of the baseband signal. In this case, $n_{\max }$ channels are estimated using the LS method, in order to remove the baseband signal and harmonics thereof from the received signal.

Widely linear digital cancellation, which takes into account IQ imbalance, was proposed in [25], where it is assumed that:

$$
r=h_{\mathrm{SI}} * x+h_{\mathrm{SI}, \mathrm{IQ}} * x^{*}+s+z,
$$

where $h_{\mathrm{SI}}$ and $h_{\mathrm{SI}, \mathrm{IQ}}$ denote the channels experienced by the baseband signal and the complex conjugate of the baseband signal, respectively. The goal becomes to jointly estimate $h_{\mathrm{SI}}$ and $h_{\mathrm{SI} \text {,IQ }}$ using the LS method.

\subsection{Joint digital cancellation of DAC non-linearities and IQ imbalance}

Ideally, we would like to perform digital cancellation based on a model that includes all non-idealities. However, due to multiple non-linearities, a full model is highly complicated. Thus, we first examine the case where the output power is low, so that it can be safely assumed that we are only ignoring very limited RF non-linearities. In this case, the resulting non-idealities model leads to a convenient cancellation method.

\subsubsection{Low RF output power}

At low RF output power, the main sources of nonidealities are the DACs and the RF mixers, which introduce non-linearities and IQ imbalance, respectively. In the general case, IQ imbalance is frequency selective, so that $\gamma_{\mathrm{Tx}}, \delta_{\mathrm{Tx}} \in \mathbb{C}^{L}$, where $L$ is the length of the impulse response. Thus, the analog RF signal $x$ is given by:

$$
x=\Re\left\{\left(\gamma_{\mathrm{Tx}} * \tilde{x}+\delta_{\mathrm{Tx}} * \tilde{x}^{*}\right) e^{j 2 \pi f_{c} t}\right\} .
$$

The transmitter amplifier operates in its linear regime so that the amplified analog RF signal, denoted by $\hat{x}$, is assumed to be an ideal amplified version of $x$, i.e., $\hat{x}=$ $\beta_{1} x$. On the receiver side, the RF mixer introduces IQ imbalance during downconversion:

$$
\begin{aligned}
\hat{r}= & \operatorname{LPF}\left\{\beta_{1}\left(\gamma_{\mathrm{Rx}} * h_{\mathrm{SI}} * \hat{x}+\delta_{\mathrm{Rx}} * h_{\mathrm{SI}}^{*} * \hat{x}^{*}\right)\right. \\
\cdot & \left.e^{-j 2 \pi f_{c} t}\right\} \\
= & \frac{\beta_{1}}{2}\left[\gamma_{\mathrm{Eq}} * \tilde{x}+\delta_{\mathrm{Eq}} * \tilde{x}^{*}\right] \\
= & \frac{\beta_{1}}{2}\left[\left(\gamma_{\mathrm{Eq}}+\delta_{\mathrm{Eq}}\right) * \sum_{m=1}^{m_{\max }} \alpha_{1, m} \Re\{x\}^{m}\right. \\
& \left.+\left(\gamma_{\mathrm{Eq}}-\delta_{\mathrm{Eq}}\right) * \sum_{m=1}^{m_{\max }} \alpha_{2, m} \Im\{x\}^{m}\right],
\end{aligned}
$$

where:

$$
\begin{aligned}
& \gamma_{\mathrm{Eq}} \triangleq \gamma_{\mathrm{Rx}} * h_{\mathrm{SI}} * \gamma_{\mathrm{Tx}}+\delta_{\mathrm{Rx}} * h_{\mathrm{SI}}^{*} * \delta_{\mathrm{Tx}}^{*}, \\
& \delta_{\mathrm{Eq}} \triangleq \delta_{\mathrm{Rx}} * h_{\mathrm{SI}}^{*} * \gamma_{\mathrm{Tx}}^{*}+\gamma_{\mathrm{Rx}} * h_{\mathrm{SI}} * \delta_{\mathrm{Tx}} .
\end{aligned}
$$

Thus, from Equation 16 we see that, for low transmit powers, the combined effect of the transmitter and receiver IQ imbalance and the transmission channel is equivalent to the effect of a single IQ imbalance with parameters $\gamma_{\mathrm{Eq}}$ and $\delta_{\mathrm{Eq}}$. Moreover, by rewriting Equation 16 as 17, it becomes clear that a form of nonlinear cancellation is required even at low output powers due to the non-linearities introduced by the DACs.

$$
\begin{aligned}
\hat{x}= & \sum_{\substack{n=1 \\
n \text { odd }}}^{n_{\max }} \beta_{n} x^{n} \\
= & \sum_{\substack{n=1 \\
n \text { odd }}}^{n_{\max }} \frac{\beta_{n}}{2^{n}}\left[\sum_{k=0}^{n}\left(\begin{array}{l}
n \\
k
\end{array}\right)\left[\left(\gamma_{\mathrm{Tx}} * \tilde{x}+\delta_{\mathrm{Tx}} * \tilde{x}^{*}\right) e^{j 2 \pi f_{c} t}\right]^{n-k}\right. \\
& \left.\times\left[\left(\gamma_{\mathrm{Tx}}^{*} * \tilde{x}^{*}+\delta_{\mathrm{Tx}}^{*} * \tilde{x}\right) e^{-j 2 \pi f_{c} t}\right]^{k}\right]
\end{aligned}
$$

We propose to cancel the IQ imbalance and DAC nonlinearities jointly, by constructing an LS-based non-linear 
cancellation scheme based on the model of Equation 17, which contains only $\Re\{x\}$ and $\mathfrak{\Im}\{x\}$ and powers thereof.

\subsubsection{Performance of digital cancellation}

In order to assess the performance of different digital cancellation mechanisms on our testbed, we conduct the following experiment. We construct 100 frames containing a $10 \mathrm{MHz} 4-\mathrm{QAM}$ modulated signal. We transmit each group of frames using transmit powers ranging from -10 up to $22 \mathrm{dBm}$. The carrier frequency is set to $2.48 \mathrm{GHz}$. We consider two antenna spacings that give us 40 and $55 \mathrm{~dB}$ of passive analog suppression. In practice, these suppression numbers are easily achievable by using a combination of passive suppression and active analog suppression, but as mentioned earlier, in our experiments, no active analog cancellation is performed to keep all signal components accessible. The digital baseband samples are recorded, and the various digital cancellation methods are applied to them off-line.

In Figure 5a, we present the mean distance of the residual self-interference signal from the measured noise floor for several digital cancellation methods with $40 \mathrm{~dB}$ of passive suppression. Since the amount of cancellation is a random variable, we also include error bars at one standard deviation from the mean. We also present the apparent noise floor at each transmit power, which stems mainly from the limited dynamic range of the ADC of the receiver. The achievable cancellation of any cancellation method is limited by this apparent noise floor, and the only way to overcome it is to increase the amount of cancellation in the analog domain. We observe that simple linear cancellation alone is insufficient even at low transmit powers, as the residual signal lies at least $7 \mathrm{~dB}$ above the noise floor. Non-linear cancellation [24] only reduces the residual self-interference by an additional $2 \mathrm{~dB}$ over the entire examined range of transmit powers. The IQ imbalance cancellation method proposed in [25] improves the obtained cancellation drastically. However, our joint cancellation method consistently outperforms all previously proposed methods as it considers both the baseband non-linearities and the IQ imbalance.

In Figure 5b, we present the mean distance of the residual self-interference signal from the measured noise floor for several digital cancellation methods with $55 \mathrm{~dB}$ of passive suppression. In this case, the apparent noise floor remains equal to the measured thermal noise floor for all considered transmit powers. Moreover, we observe that at low transmit powers, almost all cancellation methods perform equally well because the required amount of suppression is relatively low and most of the non-idealities lie below the thermal noise floor. As the transmit power is increased, however, IQ imbalance cancellation and our joint cancellation perform significantly better than linear and non-linear cancellation. It is important to note

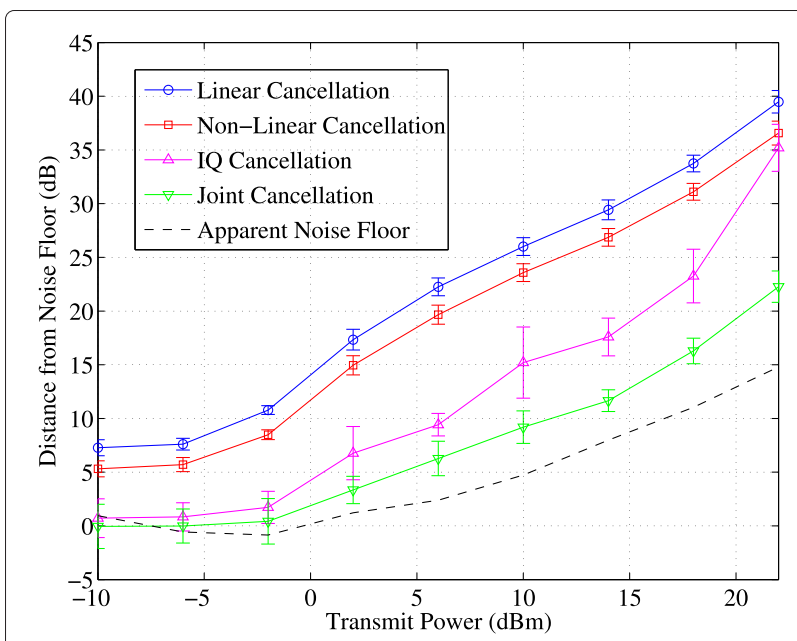

(a)

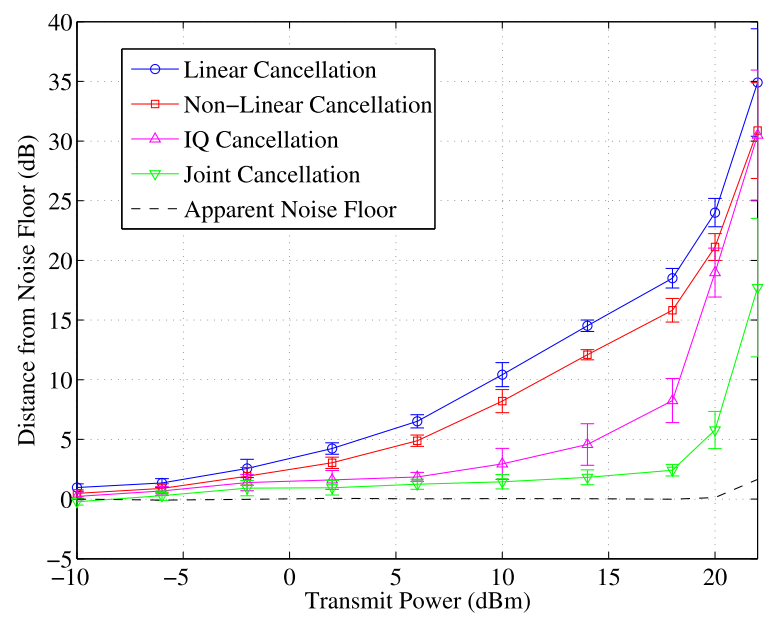

(b)

Figure 5 Distance from the thermal noise floor as a function of the transmit power. With (a) $40 \mathrm{~dB}$ analog cancellation and (b) $55 \mathrm{~dB}$ analog cancellation.

that our joint cancellation method manages to keep the residual self-interference at less than $3 \mathrm{~dB}$ above the noise floor up to a transmit power of $18 \mathrm{dBm}$. However, all cancellation methods start failing at transmit powers above $18 \mathrm{dBm}$.

\subsubsection{High RF output power}

In this section, we extend our non-idealities model to include RF non-linearities, providing some clues as to why the joint cancellation method starts failing at high transmit powers.

When the output power is high, the upconverted ana$\log$ baseband signal $x$ is unaltered with respect to the low RF power case. However, the transmitter amplifier introduces non-linearities in its output signal $\hat{x}$. In Equation 20, we use the Taylor series expansion of Equation 8 to model 
the amplified analog RF signal. At the receiver, the RF mixer introduces IQ imbalance and the received analog baseband signal $\hat{r}$ becomes:

$$
\begin{aligned}
\hat{r}= & \operatorname{LPF}\left\{\left(\gamma_{\mathrm{Rx}} * \hat{x}+\delta_{\mathrm{Rx}} * \hat{x}^{*}\right) e^{-j 2 \pi f_{c} t}\right\} \\
= & \left(\frac{\gamma_{\mathrm{Rx}}+\delta_{\mathrm{Rx}}}{2}\right) * \sum_{\substack{n=1, n \text { odd }}}^{n_{\max }} \frac{\beta_{n}}{2^{n-1}}\left(\begin{array}{c}
n \\
\frac{n-1}{2}
\end{array}\right) \\
& \times\left(\gamma_{\mathrm{Tx}} * \tilde{x}+\delta_{\mathrm{Tx}} * \tilde{x}^{*}\right)^{\frac{n+1}{2}}\left(\gamma_{\mathrm{Tx}}^{*} * \tilde{x}^{*}+\delta_{\mathrm{Tx}}^{*} * \tilde{x}\right)^{\frac{n-1}{2}}
\end{aligned}
$$

Thus, at high RF output power, the received baseband signal $r$ contains products between $\tilde{x}$ and $\tilde{x}^{*}$, or equivalently, $\Re\{\tilde{x}\}^{m}$ and $\Im\{\tilde{x}\}^{m}, m=1, \ldots, m_{\max }$, which are not contained in the model of Equation 17 and are thus not compensated for properly.

In principle, the model in Equation 22 can be used for LS-based non-linear digital cancellation similarly to the one in Equation 17. However, the number of terms in Equation 22 and, consequently, the number of channels that need to be estimated is $\left(2 m_{\max }\right)^{n_{\max }}$, which is prohibitively large even for small values of $m_{\max }$ and $n_{\max }$. For example, for $m_{\max }=n_{\max }=3$, we have $\left(2 m_{\max }\right)^{n_{\max }}=$ 216. The joint cancellation of DAC non-linearities, IQ imbalance and amplifier non-linearities remains a challenging open problem.

\section{Conclusions}

Self-interference cancellation in full-duplex systems is limited by the presence of hardware impairments in the transmitter and receiver circuits. In this paper, we provided a measurement-based study of the transceiver impairments that play a significant role in full-duplex wireless systems. Our measurements confirmed the existence of several impairments only previously considered analytically, such as phase noise and IQ imbalance, but they also demonstrated the existence of significant DAC induced baseband non-linearities. Measurements from our testbed show that our digital cancellation method that jointly takes into account DAC non-linearities and IQ imbalance achieves up to $13 \mathrm{~dB}$ more self-interference cancellation than existing digital cancellation methods.

\section{Appendix}

\section{DAC non-linearities}

Let $\omega \triangleq 2 \pi f$ denote the transmitted tone frequency so that for the ideal complex analog baseband signal we have:

$$
\tilde{x}=e^{j \omega t}=\cos (\omega t)+j \sin (\omega t)
$$

Assume that DACs are perfectly matched so that $\alpha_{1, m}=$ $\alpha_{2, m}=\alpha_{m}, m \in\left\{1, \ldots, m_{\max }\right\}$. Then, the non-ideal complex analog baseband signal with DAC induced nonlinearities is:

$$
\begin{aligned}
\tilde{x} & =\sum_{m=1}^{m_{\max }} \alpha_{m} \Re\{x\}^{m}+j \sum_{m=1}^{m_{\max }} \alpha_{m} \Im\{x\}^{m} \\
& =\sum_{m=1}^{m_{\max }} \alpha_{m} \cos ^{m}(\omega t)+j \sum_{m=1}^{m_{\max }} \alpha_{m} \sin ^{m}(\omega t) .
\end{aligned}
$$

Let $\omega_{c} \triangleq 2 \pi f_{c}$ denote the carrier frequency. Assuming an ideal RF mixer, the analog RF signal is given by:

$$
\begin{aligned}
\hat{x}= & \cos \left(\omega_{c} t\right) \sum_{m=1}^{m_{\max }} \alpha_{m} \cos ^{m}(\omega t) \\
& +\sin \left(\omega_{c} t\right) \sum_{m=1}^{m_{\max }} \alpha_{m} \sin ^{m}(\omega t) .
\end{aligned}
$$

We define $\omega_{m, k} \triangleq(m-2 k) \omega$ and $s_{m, k} \triangleq(-1)^{\left(\frac{m-1}{2}-k\right)}$. There are two cases for $m$. When $m$ is $o d d$, we have:

$$
\begin{aligned}
& \cos ^{m}(\omega t)=\frac{2}{2^{m}} \sum_{k=0}^{\frac{m-1}{2}}\left(\begin{array}{l}
m \\
k
\end{array}\right) \cos \left(\omega_{m, k} t\right), \\
& \sin ^{m}(\omega t)=\frac{2}{2^{m}} \sum_{k=0}^{\frac{m-1}{2}} s_{m, k}\left(\begin{array}{l}
m \\
k
\end{array}\right) \sin \left(\omega_{m, k} t\right) .
\end{aligned}
$$

Note that, since $\frac{m-1}{2}$ is always even, we have:

$$
s_{m, k}= \begin{cases}+1, & k \text { even, } \\ -1, & k \text { odd }\end{cases}
$$

By replacing Equations 27 and 28 in 26, we get:

$$
\begin{aligned}
\hat{x}= & \cos \left(\omega_{c} t\right) \sum_{m=1}^{m_{\max }} \frac{\alpha_{m}}{2^{m-1}} \sum_{k=0}^{\frac{m-1}{2}}\left(\begin{array}{c}
m \\
k
\end{array}\right) \cos \left(\omega_{m, k} t\right) \\
& +\sin \left(\omega_{c} t\right) \sum_{m=1}^{m_{\max }} \frac{\alpha_{m}}{2^{m-1}} \sum_{k=0}^{\frac{m-1}{2}} s_{m, k}\left(\begin{array}{c}
m \\
k
\end{array}\right) \sin \left(\omega_{m, k} t\right) \\
= & \sum_{m=1}^{m_{\max }} \frac{\alpha_{m}}{2^{m}} \sum_{k=0}^{\frac{m-1}{2}}\left(\begin{array}{c}
m \\
k
\end{array}\right)\left(1+s_{m, k}\right) \cos \left(\omega_{c} t-\omega_{m, k} t\right) \\
& +\left(1-s_{m, k}\right) \cos \left(\omega_{c} t+\omega_{m, k} t\right) .
\end{aligned}
$$

Thus, when $s_{k, m}=+1$ all $\cos \left(\omega_{c} t+\omega_{m, k} t\right)$ terms disappear. On the other hand, when $s_{k, m}=-1$, all $\cos \left(\omega_{c} t-\omega_{m, k} t\right)$ terms disappear. Thus, with ideal downconversion, the harmonics resulting from odd values of $m$ appear at frequencies $m(-1)^{\frac{m-1}{2}} \omega$ in the analog baseband. 
Let $c_{m} \triangleq \frac{1}{2^{m}}\left(\begin{array}{c}m \\ \frac{m}{2}\end{array}\right)$. When $m$ is even, we have:

$$
\begin{aligned}
& \cos ^{m}(\omega t)=c_{m}+\frac{2}{2^{m}} \sum_{k=0}^{\frac{m}{2}-1}\left(\begin{array}{l}
m \\
k
\end{array}\right) \cos \left(\omega_{m, k} t\right), \\
& \sin ^{m}(\omega t)=c_{m}+\frac{2}{2^{m}} \sum_{k=0}^{\frac{m}{2}-1} s_{m+1, k}\left(\begin{array}{c}
m \\
k
\end{array}\right) \cos \left(\omega_{m, k} t\right) .
\end{aligned}
$$

By replacing Equations 32 and 33 in 26, it can be shown similarly that no terms cancel out. Thus, with ideal downconversion, the harmonics resulting from even values of $m$ appear at both $-m \omega$ and $+m \omega$ in the analog baseband signal.

\section{Competing interests}

The authors declare that they have no competing interests.

Received: 9 December 2014 Accepted: 6 April 2015

Published online: 23 May 2015

\section{References}

1. S Chen, MA Beach, JP McGeehan, Division-free duplex for wireless applications. Electron. Lett. 34(2), 147-148 (1998)

2. A Sabharwal, P Schniter, D Guo, DW Bliss, S Rangarajan, R Wichman, In-band full-duplex wireless: challenges and opportunities. IEEE J. Sel. Areas Commun. 32(9), 1637-1652 (2014)

3. S Hong, J Brand, JI Choi, M Jain, J Mehlman, S Katti, P Levis, Applications of self-interference cancellation in $5 \mathrm{G}$ and beyond. IEEE Commun. Mag. 52(2), 114-121 (2014)

4. M Jain, JI Choi, T Kim, D Bharadia, S Seth, K Srinivasan, P Levis, S Katti, P Sinha, in Proceedings of the 17th Annual International Conference on Mobile Computing and Networking. Practical, real-time, full duplex wireless (ACM Las Vegas, NV, USA, 2011), pp. 301-312

5. M Duarte, C Dick, A Sabharwal, Experiment-driven characterization of full-duplex wireless systems. IEEE Trans. Wireless Commun.

11(12), 4296-4307 (2012)

6. W Afifi, M Krunz, in Proc. IEEE INFOCOM. Exploiting self-interference suppression for improved spectrum awareness/efficiency in cognitive radio systems (Turin, Italy, 2013), pp. 1258-1266

7. G Zheng, I Krikidis, B Ottersten, Full-duplex cooperative cognitive radio with transmit imperfections. IEEE Trans. Wireless Commun. 12(5), 2498-2511 (2013)

8. I Krikidis, C Nicosia, HA Suraweera, PJ Smith, C Yuen, Full-duplex relay selection for amplify-and-forward cooperative networks. IEEE Trans. Wireless Commun. 11(12), 4381-4393 (2012)

9. L Zhang, D Guo, Virtual full duplex wireless broadcasting via compressed sensing. IEEE/ACM Trans. Netw. 22(5), 1659-1671 (2014)

10. TRiihonen, S Werner, R Wichman, Hybrid full-duplex/half-duplex relaying with transmit power adaptation. IEEE Trans. Wireless Commun. 10(9), 3074-3085 (2011)

11. PT Hiep, R Kohno, Water-filling for full-duplex multiple-hop MIMO relay system. EURASIP J. Wireless Commun. Networking. 2014, 174 (2014)

12. G Zheng, I Krikidis, J Li, AP Petropulu, B Ottersten, Improving physical layer secrecy using full-duplex jamming receivers. IEEE Trans. Signal Process. 61(20), 4962-4974 (2013)

13. S Oashi, M Bandai, in 9th Asia-Pacific Symposium on Information and Telecommunication Technologies (APSITT). Performance of medium access control protocols for full-duplex wireless LANs (Santiago and Valparaiso, Chile, 2012), pp. 1-4

14. G Fettweis, M Lohning, D Petrovic, M Windisch, P Zillmann, W Rave, in IEEE 16th International Symposium on Personal, Indoor and Mobile Radio Communications (PIMRC). Dirty RF: a new paradigm, vol. 4 (Berlin, Germany, 2005), pp. 2347-2355

15. T Schenk, RF Imperfections in High-rate Wireless Systems: Impact and Digital Compensation. (Springer, Netherlands, 2008)
16. L Smaïni, RF Analog Impairments Modeling for Communication Systems Simulation: Application to OFDM-based Transceivers. (Wiley, Chichester, 2012)

17. S Shao, X Quan, Y Shen, Y Tang, in IEEE International Conference on Acoustics, Speech and Signal Processing (ICASSP). Effect of phase noise on digital self-interference cancellation in wireless full duplex (Florence, Italy, 2014), pp. 2759-2763

18. A Sahai, G Patel, C Dick, A Sabharwal, On the impact of phase noise on active cancelation in wireless full-duplex. IEEE Trans. Veh. Technol. 62(9), 4494-4510 (2013)

19. E Ahmed, A Eltawil, On phase noise suppression in full-duplex systems. IEEE Trans. Wireless Commun. 14(3), 1237-1251 (2015)

20. V Syrjala, M Valkama, L Anttila, T Riihonen, D Korpi, Analysis of oscillator phase-noise effects on self-interference cancellation in full-duplex OFDM radio transceivers. IEEE Trans. Wireless Commun. 13(6), 2977-2990 (2014)

21. D Korpi, T Riihonen, V Syrjala, L Anttila, M Valkama, R Wichman, Full-duplex transceiver system calculations: analysis of ADC and linearity challenges. IEEE Trans. Wireless Commun. 13(7), 3821-3836 (2014)

22. S Li, RD Murch, An investigation into baseband techniques for single-channel full-duplex wireless communication systems. IEEE Trans. Wireless Commun. 13(9), 4794-4806 (2014)

23. L Anttila, D Korpi, V Syrjala, M Valkama, in Asilomar Conference on Signals, Systems and Computers. Cancellation of power amplifier induced nonlinear self-interference in full-duplex transceivers (Pacific Grove, CA, USA, 2013), pp. 1193-1198

24. D Bharadia, E McMilin, S Katti, in Proc. ACM SIGCOMM Conference on SIGCOMM. Full duplex radios (Hong Kong, China, 2013), pp. 375-386

25. D Korpi, L Anttila, V Syrjala, M Valkama, Widely linear digital self-interference cancellation in direct-conversion full-duplex transceiver IEEE J. Sel. Areas Commun. 32(9), 1674-1687 (2014)

26. T Riihonen, R Wichman, in Asilomar Conference on Signals, Systems and Computers. Analog and digital self-interference cancellation in full-duplex MIMO-OFDM transceivers with limited resolution in A/D conversion (Pacific Grove, CA, USA, 2012), pp. 45-49

27. MA Khojastepour, S Rangarajan, in Asilomar Conference on Signals, Systems and Computers. Wideband digital cancellation for full-duplex communications (Pacific Grove, CA, USA, 2012), pp. 1300-1304

28. V Syrjala, K Yamamoto, in IEEE International Conference on Acoustics, Speech and Signal Processing (ICASSP). Sampling jitter in full-duplex radio transceivers: estimation and mitigation (Florence, Italy, 2014), pp. 2764-2768

29. V Syrjala, K Yamamoto, in IEEE 79th Vehicular Technology Conference (VTC Spring). Is sampling jitter a problem in full-duplex radio transceivers or not? (Seoul, Korea, 2014), pp. 1-5

30. E Everett, A Sahai, A Sabharwal, Passive self-interference suppression for full-duplex infrastructure nodes. IEEE Trans. Wireless Commun. 13(2), 680-694 (2014)

31. Texas Instruments ADS4246 Datasheet. http://www.ti.com/lit/ds/ symlink/ads4246.pdf

32. D Bharadia, S Katti, in 11th USENIX Symposium on Networked Systems Design and Implementation. Full duplex MIMO radios (Seattle, WA, USA, 2014), pp. 359-372

33. M Duarte, A Sabharwal, in Asilomar Conference on Signals, Systems and Computers. Full-duplex wireless communications using off-the-shelf radios: feasibility and first results (Pacific Grove, CA, USA, 2010), pp. 1558-1562

34. A Balatsoukas-Stimming, P Belanovic, K Alexandris, A Burg, in Asilomar Conference on Signals, Systems and Computers. On self-interference suppression methods for low-complexity full-duplex MIMO (Pacific Grove, CA, USA, 2013), pp. 992-997

35. National Instruments PXle-1082 User Manual. http://www.ni.com/pdf/ manuals/372752b.pdf

36. National Instruments 5791R User Manual and Specifications. http://www. ni.com/pdf/manuals/373845d.pdf

37. Mini-Circuits ZX60-272LN+ Datasheet. http://194.75.38.69/pdfs/ZX60 272LN+.pdf

38. Texas Instruments DAC3482 Datasheet. http://www.ti.com/lit/ds/ symlink/dac3482.pdf 